\title{
A Discrete Model For Pattern Formation In Volatile Thin Films
}

\author{
M. Malik-Garbi, O. Agam*
}

The Racah Institute of Physics, The Hebrew University, Jerusalem, 91904, Israel

\begin{abstract}
We introduce a model, similar to diffusion limited aggregation (DLA), which serves as a discrete analog of the continuous dynamics of evaporation of thin liquid films. Within mean field approximation the dynamics of this model, averaged over many realizations of the growing cluster, reduces to that of the idealized evaporation model in which surface tension is neglected. However fluctuations beyond the mean field level play an important role, and we study their effect on the conserved quantities of the idealized evaporation model. Assuming the cluster to be a fractal, a heuristic approach is developed in order to explain the distinctive increase of the fractal dimension with the cluster size.
\end{abstract}

Keywords and phrases: viscous fingering, diffusion limited aggregation, reaction-diffusion

Mathematics Subject Classification: 82B24, 82C24

\section{Introduction}

The processes of evaporation and dewetting of thin liquid films play an important role in modern technologies such as the fabrication of electronic chips, microfluidic devices, and biosensors. These complicated nonequilibrium processes incorporate many ingredients such as heat transport within the fluid and its surroundings, interaction between the substrate and the fluid, and the roughness of the substrate. In some cases the evaporation of thin liquid films exhibits a distinctive fingering pattern. An experimental system demonstrating such a behavior consists of a clean mica substrate covered by a thin water film[16]. The fingering instability in this system appears at the interface between two phases of the film's thickness (approximately 2 and $12 \mathrm{~nm}$ ). These two phases result from the competing van der Waals and polar surface forces between the water and the substrate[6]. A first-order phase transition between the film thicknesses is induced when changing the vapor pressure. When the evaporation rate is sufficiently small, such that changes in the film thicknesses are negligible, the evaporation dynamics is, essentially, that of the interface between the two phases.

The fingering instability observed in these dewetting experiments is similar to the one which appears in the Saffman-Taylor (ST) problem in Hele-Shaw cell[7]. In the latter case an inviscid fluid (say air) penetrates a viscous fluid (say oil) confined between two parallel plates separated by narrow gap. When pumping of the inviscid fluid is sufficiently strong to overcome the smoothing effect of the surface tension, the dynamics is characterized by repetend tip-splitting events which result in a fractal-like pattern.

\footnotetext{
${ }^{*}$ Corresponding author. E-mail: agam@phys.huji.ac.il
} 

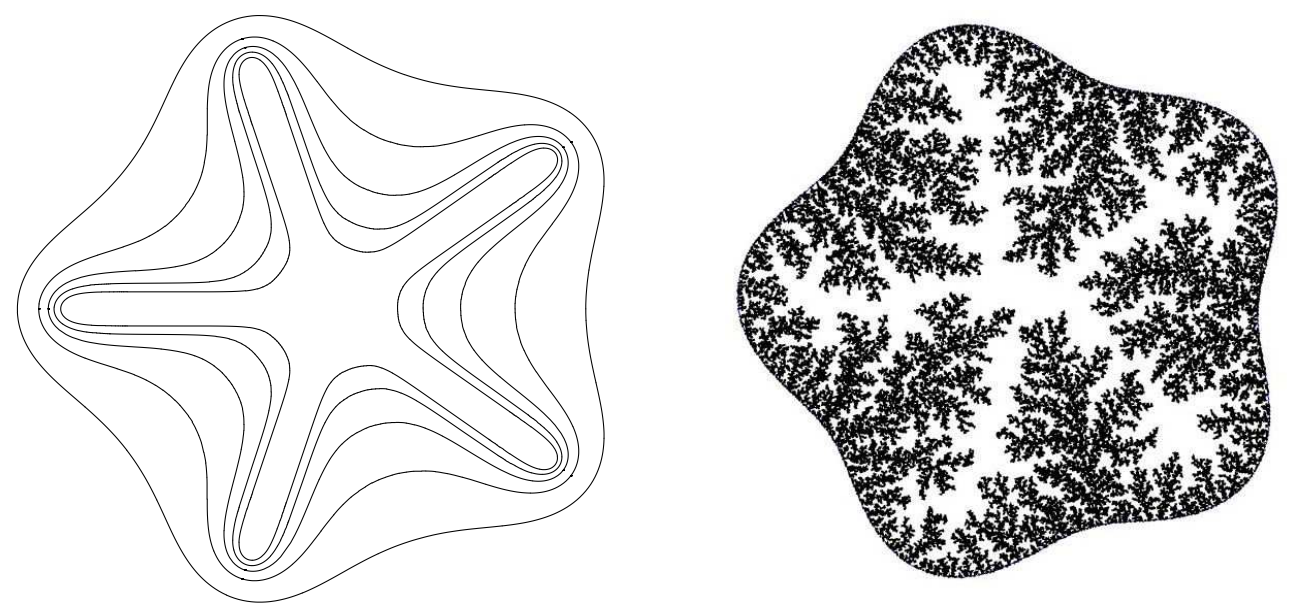

Figure 1. An example of the evolution of evaporating thin-film droplet and the corresponding discrete model. Left: snapshots (with fixed time interval) of the contour of an evaporating droplet. Right: the fractal pattern developed by the discrete model of evaporation for initial boundary which is identical to that of the left panel.

A discrete version of the ST problem is the diffusion limited aggregation (DLA) model[8].This model is defined by the following simple dynamics: A particle released far from the origin is allowed to diffuse in space. It becomes immobile when its distance from a seed particle located at the origin is smaller than some predetermined value. These two particles form the initial cluster which consequently grows into a fractal when additional diffusing particles are released one by one and become immobile as they reach the cluster. Although the model of DLA is apparently very different from the ST problem, data analysis demonstrated that their fractal properties are identical[9]. It has been also shown that averaging over many realizations of DLA patterns, in a channel geometry, reproduces a pattern similar to the ST finger developed in the same geometry[10-12]. These features suggest that the continuous ST model, whose numerical study is exceedingly difficult, may be studied using its discrete analog for which there are efficient numerical methods, e.g. iterative conformal maps [13].

The main purpose of this work is to introduce an analogous simple discrete model for evaporating thin films, to study its properties and its relation to the continuous dynamics counterpart, see Figure 1.

\section{The continuous dynamics of evaporating thin-film droplets}

The equations which govern the continuous dynamics of evaporating thin films were derived in Ref. [14] using the lubrication approximation. The dynamics is described by two equations: The first is Darcy's law, which relates the fluid velocity $\mathbf{v}$ (averaged over its profile in the direction perpendicular to the substrate) to the gradient of the pressure $P$,

$$
\mathbf{v}=-\alpha \nabla P,
$$

where $\alpha$ is a constant which depends on the fluid viscosity and the gap between the plates, while $\nabla=$ $\left(\partial_{x}, \partial_{y}\right)$ is the two dimensional gradient. The second equation is the continuity equation,

$$
\nabla \cdot \mathbf{v}=-\beta,
$$

where $\beta$ depends on the rate of evaporation and the fluid density. This equation is based on the assumption that evaporation is uniform within the droplet (i.e. the thicker film phase) while outside the droplet (the 
thinner film phase) it is essentially negligible due to the strong adhesion forces between the liquid and the substrate.

Combining the above equations one obtains that inside the droplet's domain, henceforth denoted by $\mathcal{D}$, the pressure satisfies Poisson's equation, while outside this region it is constant. Without loss of generality one may set the outside pressure to be zero, and thus

$$
\begin{gathered}
\nabla^{2} P=\gamma \text { within } \mathcal{D} \\
P=0 \text { otherwise, }
\end{gathered}
$$

where $\gamma=\beta / \alpha$. This equation describes the pressure away from the droplet's interface. Near the interface, additional degrees of freedom, such as the film height and the boundary curvature, as well as other instabilities along the droplet's rim come into play[15].

In what follows we shall neglect these effects and assume they may be incorporated, at least approximately, by a proper choice of the initial conditions. In particular we shall neglect the surface tension associated with the interface between the two film thicknesses. With this assumption the boundary condition for Eq. (2.3) is $P=0$ on the boundary of the droplet, $\partial \mathcal{D}$. This boundary moves in accordance to Darcy's law, namely its normal velocity is proportional to the normal derivative of the pressure:

$$
v_{n}=-\alpha \frac{\partial P}{\partial n} .
$$

\section{Integrability of the ideal continuous model of evaporation}

The above model for the continuous evolution of the droplet's shape is integrable in the following sense: The droplet shape may be computed exactly within some interval of time for arbitrary smooth initial conditions. This feature emerges from an infinite number of conserved quantities associated with the droplet shape. These quantities are the interior harmonic moments defined by the integrals,

$$
t_{k}=\frac{1}{\pi} \int_{z \in \mathcal{D}(t)} d x d y z^{k}
$$

where $z=x+i y$ is the complex coordinate, $k$ is a non-negative integer, and the integration is over the droplet's domain, $\mathcal{D}(t)$, whose time dependence is explicitly written here. The harmonic moments of the droplet satisfy the conservation law $[14,16]$ :

$$
t_{k}(t) \exp (\beta t)=t_{k}(0)=\text { Const. }
$$

where $\beta$ is the rate defined by the continuity equation (2.2). An example for the evolution of the a droplet is shown in the left panel of Figure 1 (for details of the calculation see [14]).

\section{A discrete model for evaporating thin-films}

A discrete model associated with the above evolution is defined by the following procedure: A particle is introduced at a random location which is uniformly distributed within a domain that is identical to the initial droplet's shape. The particle diffuses until it gets close enough to the boundary (within some fixed cutoff distance). At this point the particle becomes immobile and part of the exterior domain. The process is repeated with additional particles so that the domain boundary develops into a fractal-like pattern. An example for such pattern is depicted in the right panel of Figure 1.

To clarify the relation between the discrete model of evaporation and its continuous counterpart described by Eqs. (2.4) and (2.2) it is instructive to represent the discrete model as a reaction-diffusion 
problem. For this purpose we discretize space and denote its lattice points by the index $j$. We introduce two types of particles: Diffusing $A$ particles, and immobile $B$ particles. The latter represent the growing cluster. An $A$ particle is born randomly (with a uniform distribution) at some unoccupied site and is allowed to diffuse until it reaches a neighboring $B$ particle. At this point it converts to $B$. The reaction-diffusion processes associated with this dynamics are described by:

$$
\begin{gathered}
\emptyset \stackrel{\mu}{\longrightarrow} A_{j} \\
A_{j} \stackrel{\nu}{\longrightarrow} A_{\tilde{j}} \\
A_{j}+B_{\tilde{j}} \stackrel{\lambda}{\longrightarrow} B_{j}+B_{\tilde{j}}
\end{gathered}
$$

The first line represents the process of a birth of $A$ particle at some arbitrary location denoted by the subscript $j$. The second line describes the random hopping of $A$ article from the site $j$ to a nearest neighbor site $\tilde{j}$, and the third process accounts for the adhesion of $A$ particle to the cluster of immobile $B$ particles: Once $A$ reaches a nearest neighbor site occupied by $B$ particle it converts to $B$. The parameters that appear above the arrows represent the rates associated with each one of the process. Our discrete evaporation model is achieved in the limit where the birth rate of new particles, $\mu$, is sufficiently low so that at any moment of time there is at most one $A$ particle in the system, and the adhesion rate is large enough so that the particle sticks to the cluster before it has a chance to hop away, $\lambda / \nu \gg 1$. For simplicity, the reaction diffusion process describes by (4.1) correspond to on-lattice dynamics. It can be straightforwardly generalized to off-lattice dynamics by assuming that the size of the particles is large compared to the lattice constant and assuming that particles cannot overlap.

The dynamics of reaction diffusion systems are described by the master equations which determine the evolution of the probability of the various configurations of the system. Let us denote this probability by $P_{\mathbf{n}, \mathbf{m}}$, where the configuration of the system is specified by the vectors $\mathbf{n}=\left(n_{1}, n_{2}, \cdots\right)$ and $\mathbf{m}=$ $\left(m_{1}, m_{2}, \cdots\right)$ in which $n_{j}$ and $m_{j}$ are the numbers of $A$ and $B$ particles at site $j$, respectively.

The master equations may be represented in the form of a Schrödinger equation in imaginary time [17-20]. For this purpose one defines the wave function,

$$
|\psi\rangle=\sum_{\mathbf{n}, \mathbf{m}}^{\infty} P_{\mathbf{n}, \mathbf{m}}|\mathbf{n}, \mathbf{m}\rangle,
$$

and the annihilation and creation operators, $\hat{a}_{j}$, and $\hat{a}_{j}^{\dagger}$, of $A$ particle at site $j$, and similarly the annihilation and creation operators of $B$ particles: $\hat{b}_{j}$, and $\hat{b}_{j}^{\dagger}$. These operators satisfy the Bose commutation relations $\left[\hat{a}_{j}, \hat{a}_{j^{\prime}}^{\dagger}\right]=\delta_{j, j^{\prime}}$, and $\left[\hat{b}_{j}, \hat{b}_{j^{\prime}}^{\dagger}\right]=\delta_{j, j^{\prime}}$. With these definitions one can verify that the master equations take the form:

$$
\frac{d}{d t}|\psi\rangle=-H|\psi\rangle
$$

with the Hamiltonian:

$$
H\left[\hat{a}_{j}, \hat{a}_{j}^{\dagger}, \hat{b}_{j}, \hat{b}_{j}^{\dagger}\right]=\sum_{j, \tilde{j}} \mu\left(1-\hat{a}_{j}^{\dagger}\right)+\nu \hat{a}_{j}^{\dagger}\left(\hat{a}_{j}-\hat{a}_{\tilde{j}}\right)+\lambda\left(\hat{b}_{j}^{\dagger} \hat{a}_{\tilde{j}}^{\dagger}-\hat{b}_{j}^{\dagger} \hat{b}_{\tilde{j}}^{\dagger}\right) \hat{a}_{\tilde{j}} \hat{b}_{j} .
$$

Thus the time evolution of the wave function is given by $|\psi(t)\rangle=U(t)|\psi(0)\rangle$, where $\psi(0)$ is the initial wave function, and $U(t)$ is the propagator of the system for time $t$, i.e.

$$
U(t)=\mathcal{T} \exp \left\{-\int_{0}^{t} d \tau H(\tau)\right\},
$$

$\mathcal{T}$ being the time ordering operator[21]. 
To make sure that the evolution of the system starts within some predefined domain shape $\mathcal{D}$, one should choose the initial wave function such that there are no $A$ particles in the system and $B$ particles are located along the boundary domain $\partial \mathcal{D}$. Thus the initial wave function is

$$
|\psi(0)\rangle=\prod_{q \in \partial \mathcal{D}} b_{q}^{\dagger}|0\rangle
$$

where $|0\rangle$ denotes the vacuum state with no particles in the system.

The structure of the above solution calls for representation as a field integral. Introducing the complex functions of time, $a_{j}(\tau)$ and $b_{j}(\tau)$, and their complex conjugates, $\bar{a}_{j}(\tau)$ and $\bar{b}_{j}(\tau)$, the propagator may be written in the form:

$$
U(t)=\int d \eta e^{-S}
$$

where

$$
S=\int_{0}^{t} d \tau\left(\sum_{j}\left(\bar{a}_{j} \partial_{\tau} a_{j}+\bar{b}_{j} \partial_{\tau} b_{j}\right)+H\left[a_{j}, \bar{a}_{j}, b_{j}, \bar{b}_{j}\right]\right) .
$$

is the action, and $d \eta=\prod_{\tau, j}\left[d^{2} a_{j}(\tau) d^{2} b_{j}(\tau)\right]$ is the measure of the integral where $d^{2} z=(d \operatorname{Re} z d \operatorname{Im} z) / \pi$.

The average probability of finding a $B$ particle at site $m$ and time $t^{\prime}$ is obtained by averaging $b_{m}\left(t^{\prime}\right)$ with respect to the above action with the initial wave function (4.6):

$$
\left\langle b_{m}\left(t^{\prime}\right)\right\rangle=\int d \eta e^{-S} b_{m}\left(t^{\prime}\right) \prod_{q \in \partial \mathcal{D}} \bar{b}_{q}(0)
$$

This average should be interpreted as an average over a large number of realizations of the discrete model dynamics.

The configurations of fields that influence mostly the evolution are associated with the saddle points of the integral (4.7). The corresponding saddle point equations are obtained from variation of the action $S$ with respect to the fields. Variation of $S$ with respect to $\bar{a}_{j}$ and $\bar{b}_{j}$ lead to equations which are satisfied by $\bar{a}_{j}=\bar{b}_{j}=1$ at any lattice point $j$. Variations with respect to $a_{j}$ and $b_{j}$ result in the equations:

$$
\begin{aligned}
\partial_{\tau} a_{j} & =\mu+\sum_{\tilde{j}} \nu\left(a_{\tilde{j}}-a_{j}\right)-\lambda a_{j} b_{\tilde{j}} \\
\partial_{\tau} b_{j} & =\lambda \sum_{\tilde{j}} a_{j} b_{\tilde{j}}
\end{aligned}
$$

where as before $\tilde{j}$ denote a nearest neighbor site of $j$.

The continuum limit of these saddle point equations is obtained when the spatial changes of the fields are assumed to be very slow compared to the lower distance cutoff of the discretized space, $l_{0}$. In this limit the saddle point equations converge to rate equations for the densities of $A$ and $B$ particles.

In particular, away from the $B$ cluster (i.e. where $b_{j}=0$ ) Eq. (4.9) reduces to $\partial_{t} a=D \nabla^{2} a+\mu$, where $D=\nu l_{0}^{2}$ is diffusion constant of the $A$ particles. In the limit where birth rate is sufficiently small such that the mean time between birth events is larger than the time that takes for a particle to diffuse across the system, the time dependence may be neglected and one obtains:

$$
-D \nabla^{2} a=\mu .
$$

Consider now the case were the $A$ particle is near the cluster of $B$ particles, i.e. $b_{j} \neq 0$ in Eq. (4.9). Imposing the assumption that the conversion rate from $A$ to $B$ is very large compared to the hopping 

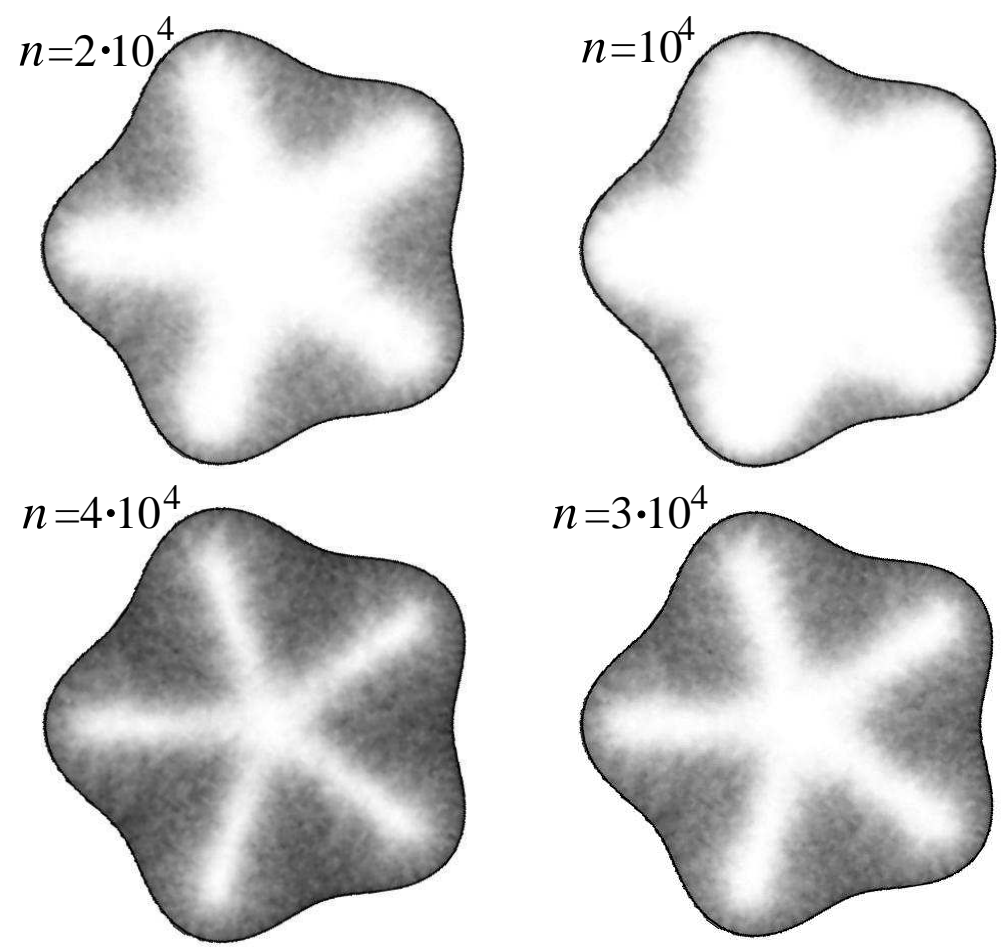

FiguRE 2. Density plots of averages over 80 realization of the discrete evaporation model with initial conditions as in Figure 1. $n$ denotes the number of particles in the developing cluster.

rate, $\lambda \gg \nu$, we obtain that on the boundary of the $B$ cluster $a=0$. Finally, taking this boundary condition into account, the continuous limit of Eq. (4.10) implies that the boundary of the $B$ cluster advances with normal velocity:

$$
v_{n}=\lambda l_{0}^{2} \frac{\partial a}{\partial n} .
$$

Thus identifying of the pressure $P$ with $-a$ we see that Eq. (4.11) and Eq. (4.12) are equivalent to Poisson equation (2.3) (with the correct boundary conditions), and Darcy's law (2.4), respectively.

\section{Fluctuations}

The result of the previous section implies that, within the saddle point approximation, the average of the probability to find a $B$ particle in a given point in space follows the evolution of the continuum dynamics of the evaporating thin film. One of the consequences of this property is that the average of the probability to find a $B$ particle in a given point in space follows the evolution of the continuum dynamics of the evaporating thin film.

However, the saddle point approximation is not justified by the existence of some large parameter, and therefore fluctuations are important. Indeed these fluctuations play an essential role in developing a fractal-like structures in the discrete evaporation model. The purpose of this section is to study this feature.

An example for the structures obtained by averaging over many realizations of the discrete evaporation model is depicted in Figure 2. This figure presents the average density of $B$ particles obtained from 


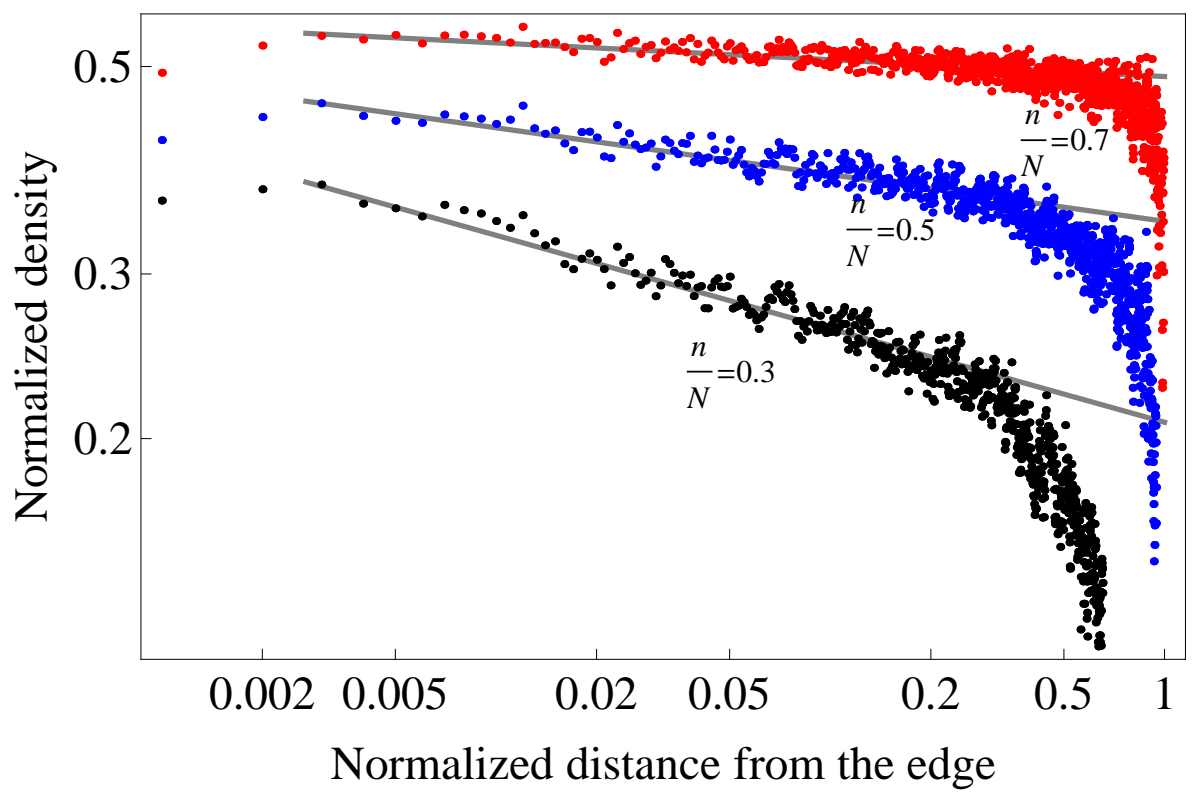

Figure 3. (color online) A log-log plot of the density of particles versus the distance from the edge of a circular domain for three different coverage rations, $n / N$. The gray lines represent power-law fits with a decaying power which decrease as the coverage ratio increases.

80 independent realizations starting from the initial boundary shape similar to that shown in Figure 1. These plots demonstrate the close relation between the discrete model and the evolution of the continuous model shown in the left panel of Figure 1. Notice, however, that within the saddle point (or mean field) approximation the density of the $B$ particles within the occupied regions should be constant, while here it decreases from the boundary inwards. This is a manifestation of the large fluctuations in the particle density as demonstrated in the right panel of Figure 1.

In order to understand the behavior of the density of the developing structures as function of the distance from the boundary, we considered a circular domain with capacity of having $N=2.26 \cdot 10^{6}$ particles, and computed the density of the particles. A log-log plot of this density as function of the distance from the circumference is plotted in Figure 3 for several covering ratios, $n / N$. This plot shows that within a large intermediate regime, which is not too close to the circumference or to the center of the circle, the density decreases as a power law with a power that decreases monotonically with the covering ratio $n / N$. This behavior (which is similar to DLA) suggests that the cluster is a fractal. Moreover the change in the power exponent, as function of the covering ratio, implies a change in the fractal dimension of the cluster, as will be discussed in the next section.

Additional quantities which demonstrate the link between the continuous model and the discrete model of evaporation are the harmonic moments. For the discrete model these harmonic moments may be represented in the form:

$$
t_{k}(t)=t_{k}(0)-\frac{1}{\pi} \sum_{j \in B(t)} l_{0}^{2} z_{j}^{k},
$$

where $t_{k}(0)$ is the harmonic moment of the initial domain shape, the sum $j$ is over the $b$ particles at time $t$, the size of each particle is $l_{0}^{2}$, and its position is defined by the complex coordinate $z_{j}=x_{j}+i y_{j}$. The average of the harmonic moment can be obtained by evaluating the average of $\sum_{j} l_{0}^{2} z_{j}^{k} b_{j}(t)$. Therefore 


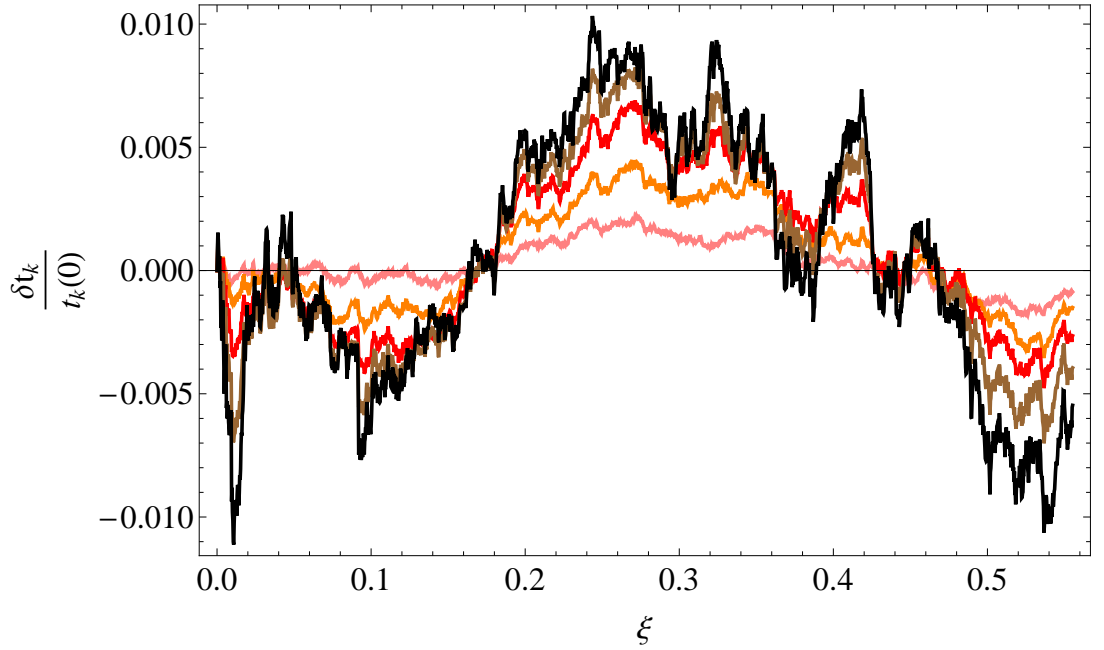

FiguRE 4. (color online) The relative fluctuation of the even harmonic moments as function of the coverage ratio for particular realization of a cluster grown in an ellipse whose aspect ratio is $1 / 2$. The various lines correspond to $k=2,4,6,8$, and 10 (colors: pink, orange, red, brown, and black respectively).

within the saddle point approximation, where the cluster of $b$ particles evolves according to the continuous dynamics, the average harmonic moments follow the dynamics dictated by the continuum evaporation model, namely

$$
\left\langle t_{k}(t)\right\rangle \approx t_{k}(0)(1-\xi)
$$

where

$$
\xi=\frac{\mathcal{A}}{\mathcal{A}_{0}}
$$

is the ratio of the cluster coverage area $\mathcal{A}$ to the total area of the initial domain, $\mathcal{A}_{0}$. Here, in order to express the harmonic moments in terms of $\xi$, we made use of the fact that the zeroth harmonic moment of the cluster, which is the area of unoccupied sites, decays exponentially with time at precisely the same rate of all other harmonic moments (3.2).

In order to see to what extent the ensemble average of the harmonic moments characterizes the behavior of some particular realization of the dynamics, we have simulated the cluster growth within an elliptical domain with aspect ratio of $1 / 2$. In this case the odd harmonic moments vanish while the even ones can be computed using definition (3.1), and the result is:

$$
t_{2 k}(0)=c_{k}\left(\frac{\mathcal{A}_{0}}{\pi}\right)^{k+1},
$$

where $\mathcal{A}_{0}$ is the area of the ellipse. The constants $c_{k}$ depend on the aspect ratio of the ellipse, and for the case in which it is $1 / 2: c_{0}=1, c_{1}=3 / 8, c_{2}=9 / 32, c_{3}=135 / 512, c_{4}=567 / 2048$, and $c_{5}=5103 / 16384$. Beyond $k=10$ these constants grow approximately factorially with $k$.

The relative fluctuations, $\delta t_{k} / t_{k}(0)=\left(\left|t_{k}\right|-\left\langle t_{k}\right\rangle\right) / t_{k}(0)$, of the leading harmonic moments of a particular realization of a cluster grown in an ellipse, are depicted in Figure 4. From this figure it is evident that the fluctuations of the low harmonic moments are small compared to the average. In other words they are self-averaging and to a good approximation behave according to (5.2). 
To appreciate the meaning of the above results let us estimate the fluctuations of the harmonic moments of a circular domain for which the averages of all harmonic moments vanish. For simplicity we assume that the covering ratio, $\xi$ is close to $1 / 2$ namely the cluster extends throughout the circle. Furthermore, we shall assume that (the connected part of) the two point correlation of the particles is characterized by a fractal-like behavior, namely it decays as a power law,

$$
C(r) \simeq C_{0}\left(\frac{1}{r}\right)^{\zeta}
$$

where $C_{0}$ is some constant which depends on $\zeta$. With these assumptions the variance of the absolute value of the $k$-th harmonic moment is given by the integral:

$$
\left\langle\left|\delta t_{k}\right|^{2}\right\rangle \simeq \frac{1}{\pi^{2}} \int d^{2} r d^{2} r^{\prime} C\left(\left|\mathbf{r}-\mathbf{r}^{\prime}\right|\right)\left(r^{\prime} r\right)^{k} e^{i k\left(\theta-\theta^{\prime}\right)} .
$$

Here $\mathbf{r}=r \exp (i \theta)$ and $\mathbf{r}^{\prime}=r^{\prime} \exp \left(i \theta^{\prime}\right)$ denote complex position vectors, and the integrals extends over the whole cluster.

Assuming $\zeta$ to be small compared to one, we expand the above integral to leading order in $\zeta$ and obtain:

$$
\left\langle\left|\delta t_{k}\right|^{2}\right\rangle \simeq-\frac{1}{\pi} \zeta C_{0} \int_{0}^{R} d r^{\prime} \int_{0}^{R} d r^{\prime}\left(r^{\prime} r\right)^{k} \int_{-\pi}^{\pi} d \theta \ln \left(r^{2}+r^{\prime 2}-2 r^{\prime} r \cos \theta\right) \exp (i k \theta)
$$

where $R$ is the radius of the circular domain. This integral can be evaluated exactly, see Appendix. The result is

$$
\left\langle\left|\delta t_{k}\right|^{2}\right\rangle \simeq \frac{C_{0} \zeta}{k(k+1)(k+2)}\left(\frac{A_{0}}{\pi}\right)^{k+2}
$$

Thus the normalized fluctuation of the $k$-th harmonic moment (to the leading order in $\zeta$ ), decays with $k$ as

$$
\delta \bar{t}_{k} \simeq\left(\left(\frac{\pi}{A_{0}}\right)^{k+2}\left\langle\left|\delta t_{k}\right|^{2}\right\rangle\right)^{1 / 2} \propto \frac{1}{\sqrt{k(k+1)(k+2)}} .
$$

In Figure 5, we present the results of a numerical calculation of the harmonic moments for a cluster in a circular domain with capacity of $2.26 \cdot 10^{6}$ particles. The average of $\left\langle\left|\delta t_{k}\right|^{2}\right\rangle$ is performed over different values of the covering ratio, between 0.35 and 0.45 . Formula (5.9) is presented by the solid line. It shows a reasonable agreement between the numerical results and the theoretical estimation.

Notice that unlike the elliptical domain where $\delta t_{k}$ increases with $k$, in the circular domain the fluctuations decrease. Thus the fluctuations in the harmonic moments are strongly affected by the shape of the boundary. Similar analysis shows that the cross correlations of harmonic moments, $\left\langle\delta t_{k} \delta t_{k^{\prime}}\right\rangle$, are also governed by the shape of the boundary of the system. The manifestation of these cross correlations can be clearly observed in Figure 4.

\section{The evolution of the fractal dimension.}

One of the basic characteristic of the viscous fingering is the fractal dimension of the corresponding patterns. The construction of an exact analytic scheme for calculating the fractal dimension of ST problem is a prominent challenge. In fact, even its numerical calculation is exceedingly difficult because the equations which govern the ST evolution are very stiff, and in the presence of surface tension the problem becomes chaotic. Thus apart from a rather limited amount of experimental data, our main 


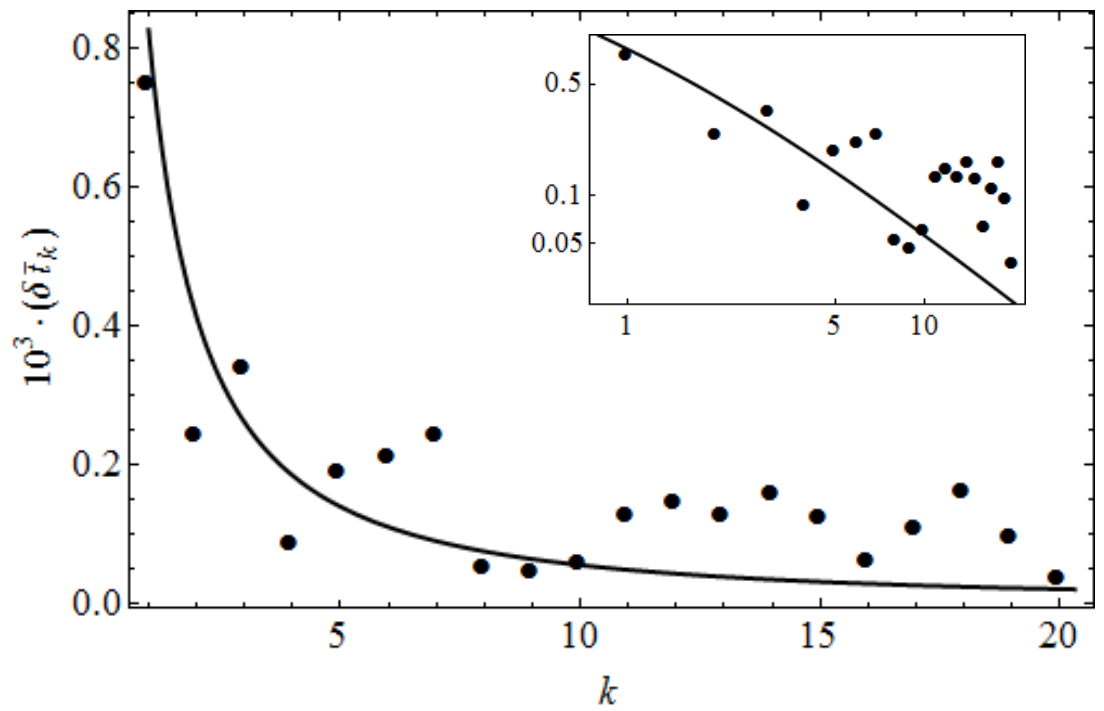

FiguRE 5. The normalized fluctuation of the first 20 harmonic moments (dots), and the theoretical estimate (solid line) described by formula (5.9). The inset shows the same data on a log-log plot.

knowledge about the fractal dimension of the ST problem is based on its relation to its discrete analog the DLA. It is believe that both systems belong to the same universality class[9].

Since similar feature might characterize the relation between the discrete and the true evolution of evaporating thin films, we turn our attention to study the fractal dimension of patterns generated by the discrete evaporation model. Based on the results for the density of the clusters (see Figure 3), and the harmonic moments (Figure 4), in what follows, we shall assume that the cluster can be considered to be a fractal whose local properties are approximately the same at any point within the bulk. This is an approximation since as can be seen from Figure 3, the dynamic range in which the cluster exhibits power law decay is limited to an annulus whose outer radius is smaller than the circle radius, and its inner radius is finite. This is because, at the beginning of the growing process, the clusters develop independently at different places along the circumference, and only later when the fingers become long enough they start to interact. DLA exhibits a similar behavior: It may be considered to be a fractal only within an annulus which is far enough from the center and from the edge of the cluster. Yet, as the cluster grows, the ratio of the number of particles within this dynamic range, to the total number of particles in the cluster, approaches one.

The fractal dimension of the cluster, $D_{f}$, is calculated using box counting, namely

$$
D_{f}=\frac{1}{\log 2} \frac{d \log N_{q}}{d q}
$$

where $N_{q}$ is the number of boxes occupied by $B$ particles, whose edge size equals to $2^{-q} L$ and $q$ is limited from above by the condition that this size is much larger than that of an individual particle. Our numerical calculation is performed for a cluster that grew in a circular domain with capacity of having $2.26 \cdot 10^{6}$ particles. The derivative with respect to $q$ is calculated from the slope of a quadratic fit to $\log \left(N_{q}\right)$ at three values of $q$. This fit is obtained from values of $N_{q}$ associated with squares of the sizes $3 \cdot 2^{-q} R$, where $R$ is the radius of the circle, and $q=8,9$ and 10 . The result of this calculation is represented by the dots in Figure 6 . It illustrates the manner by which the fractal dimension, $D_{f}$, evolves as the coverage ratio, $\xi$ (defined by Eq. (5.3)), increases from zero to one. When $\xi \ll 1$, there are 


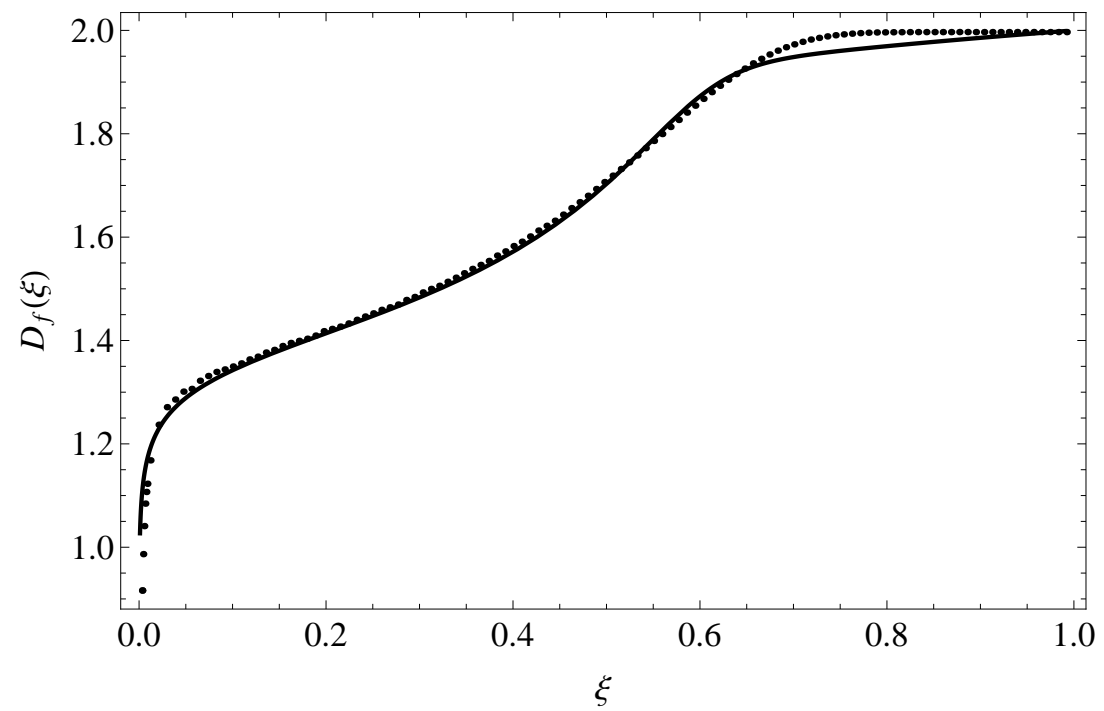

Figure 6 . The evolution of the fractal dimension as the coverage ratio of the cluster increases from zero to one. The dots represent the numerical results, while the solid line is the theoretical approximation.

only few $B$ particles scattered around the boundary of the domain, and therefore the fractal dimension approaches zero. In the other extreme, of full coverage $\xi \rightarrow 1$, the fractal dimension obviously approaches 2. Between these limits there is a monotonic growth with a steep initial slope, and a distinctive shoulder.

In order to understand this behavior we developed a heuristic approach based on the assumption that the change in the fractal dimension, when additional particles are added to the cluster, comes from two types of contributions: The first type is when the $A$ particle is born in the close vicinity of the $B$ cluster, i.e. in one of the unoccupied sites in the domain that envelopes the cluster. The second contribution comes from $A$ particles that are born far from the cluster. The effect of particles belonging to the first group is to increase the fractal dimension of the cluster without changing its typical size, while the contribution from the second group is associated with a change of the cluster's size.

To begin with, let us focus our attention on the domain that envelopes the cluster. In the case of an isotropic fractal (such as DLA) made of $n$ particles (of identical size) its area can be estimated using the radius of gyration of the cluster:

$$
R_{g}(n) \propto l_{0} n^{\frac{1}{D_{f}}}
$$

where $l_{0}$ denotes the size of each particle. Thus the effective area of the enveloping domain is proportional to the square of the gyration radius: $l_{0}^{2} n^{\frac{2}{D_{f}}}$.

However, this result applies for simple isotropic cluster, while in our system the fractal develops simultaneously at several places along the boundary of the system. The area occupied by the clusters developed according the discrete evaporation model may be written in the form

$$
\mathcal{A} \approx l_{0}^{2} m\left(\frac{n}{m}\right)^{\frac{2}{D_{f}}}
$$

where $m$ is the number of different clusters developed along the boundary of the system.

In order to identify the value of $m$ let us assume that the clusters are approximately of the same size, and for simplicity, choose the system to be a cylinder whose circumference and length both equal $L$. We denote by $w$ the total width of the cluster developed along the two boundaries of the cylinder. This width 
is approximately equal to the gyration radius of a cluster containing $n / m$ particles, i.e. $w \approx l_{0}(n / m)^{1 / D_{f}}$. The total area of the domain that envelopes the cluster is $\mathcal{A}=w L$ and from Eq. (6.3) we obtain:

$$
l_{0}^{2} m\left(\frac{n}{m}\right)^{\frac{2}{D_{f}}} \approx w L \approx l_{0}\left(\frac{n}{m}\right)^{\frac{1}{D_{f}}} L .
$$

¿From here we have $m=\left(L / l_{0}\right)^{D_{f} /\left(D_{f}-1\right)} n^{1 /\left(1-D_{f}\right)}$, and substituting this result in $(6.3)$ we get that the area of the enveloping domain is approximately:

$$
\mathcal{A} \approx l_{0}^{2}\left(\frac{L}{l_{0}}\right)^{\frac{D_{f}-2}{D_{f}-1}} n^{\frac{1}{D_{f}-1}}
$$

Now if we assume that the total area of the system, $L^{2}$, may be filled by $N$ particles, namely $L^{2}=N l_{0}^{2}$, than the ratio of the area occupied by the fractal to the total area of the system is:

$$
r\left(\xi, D_{f}\right)=\frac{\mathcal{A}}{L^{2}} \approx(\sqrt{N})^{\frac{2-D_{f}}{D_{f}-1}} \xi^{\frac{1}{D_{f}-1}},
$$

where

$$
\xi=\frac{n}{N} .
$$

Of course, since we did not take into account the interactions between different developing clusters along the boundary, this result is only an approximation.

The fraction of the unoccupied sites within the domain which envelopes the cluster to the total area of the system is $r-\xi$, while the fraction of the area outside the enveloping domain (which by definition is unoccupied) to the total area of the system is $1-r$. Since $A$ particles are born uniformly within the unoccupied area, the probability that an $A$ particle is born in one of the unoccupied sites within the enveloping domain is:

$$
P_{i n}=\frac{r-\xi}{(r-\xi)+(1-r)}=\frac{(\sqrt{N})^{\frac{2-D_{f}}{D_{f}-1}} \xi^{\frac{1}{D_{f}-1}}-\xi}{1-\xi},
$$

while the probability that an $A$ particle is born outside this domain is clearly $P_{\text {out }}=1-P_{\text {in }}$.

Consider now the change in the fractal dimension for each one of these cases. Let us examine first the case where the $A$ particle is born in one of the unoccupied sites of the enveloping domain. In this case we shall assume that the area of the enveloping domain remains intact, and therefore in order to accommodate the new particle the fractal dimension must increase. Thus if we denote by $\Delta D_{f}$ the increase in fractal dimension, and by $\Delta \xi=1 / N$ the effective change in $\xi$ due to the addition of one particle, we require that

$$
r\left(\xi+\Delta \xi, D_{f}+\Delta D_{f}\right)=r\left(\xi, D_{f}\right) .
$$

Expanding to linear order in $\Delta D_{f}$ and $\Delta \xi$ we obtain that the change in the fractal dimension due to particles born close to the fractal is

$$
\left(\frac{D_{f}}{d \xi}\right)_{i n}=\frac{\Delta D_{f}}{\Delta \xi}=\frac{D_{f}-1}{\xi \ln (\sqrt{N} \xi)}
$$

Let us consider now the change in the fractal dimension when the $A$ particle is born away from the fractal. In this case we shall assume that the effect manifests itself in a change of the radius of gyration by an amount of

$$
\Delta r *=\left.\frac{\partial r\left(\xi, D_{f}\right)}{\partial \xi}\right|_{D_{f}=D_{f}^{*}} \Delta \xi
$$


where $D_{f}^{*}$ is some unknown parameter which governs the growth of the cluster in the case where the particles come from a far distance. To this value of fractal dimension the system would have flow if all $A$ particles where born far away from the cluster. The similarity between this situation and the dynamics of DLA suggests that $D_{f}^{*}$ should be close to the fractal dimension of DLA. To identify the change in the fractal dimension due to this contribution we demand that

$$
r\left(\xi+\Delta \xi, D_{f}+\Delta D_{f}\right)-r\left(\xi, D_{f}\right)=\Delta r^{*} .
$$

Expanding the left hand side of this equation to linear order $\Delta \xi$ and $\Delta D_{f}$, and solving for $\Delta D_{f} / \Delta \xi$, we obtain

$$
\left(\frac{D_{f}}{d \xi}\right)_{\text {out }}=\left(\frac{D_{f}}{d \xi}\right)_{\text {in }}\left(1-\frac{D_{f}-1}{D_{f}^{*}-1}(\sqrt{N \xi})^{\frac{1}{1-D_{f}}-\frac{1}{1-D_{f}^{*}}}\right) .
$$

Collecting the above results we arrived at the following nonlinear equation for the evolution of the fractal dimension:

$$
\frac{D_{f}}{d \xi}=P_{i n}\left(\frac{D_{f}}{d \xi}\right)_{i n}+\left(1-P_{\text {in }}\right)\left(\frac{D_{f}}{d \xi}\right)_{\text {out }} .
$$

The numerical solution of this equation is presented by the solid line in Figure 6 . Here we treated $D_{f}^{*}$ as a fitting parameter, and choose the initial conditions to fit the data points around $\xi=0.1$ (at $\xi=0$ the equation is singular and the solution is very sensitive to the precise value of the initial condition). Our best fit is obtained for $D_{f}^{*}=1.76$. It is not far from the fractal dimension of DLA which is believed to be 1.71 .

\section{Summary}

In this work we introduced a discrete model for the evolution of evaporating thin films. We showed that on mean field level an average over many realization of the cluster growth reproduce the continuous dynamics, but, as for DLA, fluctuations play an important role. We showed that the low harmonic moments of the system have the property of self averaging, and that the magnitude of their fluctuations depends on the boundary shape. In particular, the relative fluctuations of the low harmonic moments, in an ellipse, increase with the order of the moment, while in a circular domain they decrease.

Unlike DLA, the fractal dimension of the clusters in the discrete evaporation model change with time, and grows monotonically from zero (when few particles are scattered along the boundary) to two (when particles fill the whole system). We showed that the distinctive dependence of the fractal dimension on the coverage ratio may be heuristically explained as the result of two competing contributions. The first is associated with particles that are born away from the boundary of the cluster. This contribution attracts the fractal dimension to a value similar to that of DLA. The second contribution comes from particles that are born in the close to cluster. It becomes dominant as the fractal grows, and eventually brings the fractal dimension value to two.

Acknowledgements. We thank Eldad Bettelheim for useful discussions. This research has been supported in part by the Israel Science Foundation (ISF) under Grant No. 9/09.

\section{Appendix}

In this appendix the integral (5.7) is evaluated. Writing the logarithm in the form

$$
\ln \left(r^{2}+r^{\prime 2}-2 r^{\prime} r \cos \theta\right)=\ln \left(r^{2}+r^{\prime 2}\right)+\ln \left(1-\frac{2 r^{\prime} r}{r^{2}+r^{\prime 2}} \cos \theta\right),
$$


and integrating by parts we obtain:

$$
\left\langle\left|\delta t_{k}\right|^{2}\right\rangle=\frac{1}{\pi} \zeta C_{0} \int_{0}^{R} d r^{\prime} \int_{0}^{R} d r^{\prime}\left(r^{\prime} r\right)^{k+1} \int_{-\pi}^{\pi} d \theta \frac{\mu \sin \theta}{1-\mu \cos \theta} \frac{\exp (i k \theta)}{i k}
$$

where

$$
\mu=\frac{2 r^{\prime} r}{r^{2}+r^{\prime 2}}
$$

Changing variables to $z=\exp (i \theta)$, the integral over $\theta$ transforms into a contour integral whose calculation using the residue theorem is straightforward:

$$
I=\int_{-\pi}^{\pi} d \theta \frac{\mu \sin \theta}{1-\mu \cos \theta} \frac{\exp (i k \theta)}{i k}=\frac{1}{i k} \oint \frac{z^{k+1}-z^{k-1}}{z^{2}-(2 / \mu) z+1}=\frac{2 \pi}{k}\left(\frac{1-\sqrt{1-\mu^{2}}}{\mu}\right)^{k},
$$

and using definition (8.3) we obtain:

$$
I=\frac{2 \pi}{k}\left\{\begin{array}{l}
\left(r / r^{\prime}\right)^{k} \text { for } \quad r^{\prime}>r \\
\left(r^{\prime} / r\right)^{k} \text { for } \quad r>r^{\prime}
\end{array}\right.
$$

Finally, substituting this result in (8.2), and performing the integrals over $r$ and $r^{\prime}$, leads to (5.8).

\section{References}

[1] M. Elbaum, S.G. Lipson. How does a thin wetted film dry up? Phys. Rev. Lett., 72 (1994), $3562-3565$.

[2] S.G. Lipson. Pattern formation in drying water films. Physica Scripta, T67 (1996), 63-66.

[3] I. Leizerson, S.G. Lipson, A.V. Lyushnin. Finger instability in wetting-dewetting phenomena. Langmuir, 20 (2004), 291-194.

[4] S. G. Lipson. A thickness transition in evaporating water films. Phase Transitions, 77 (2004), 677-688.

[5] I. Leizerson, S.G. Lipson. How does a thin volatile film move? Langmuir, 20 (2004), 8423-8425.

[6] N. Samid-Merzel, S.G. Lipson, D.S. Tannhauser. Pattern formation in drying water films. Phys. Rev., E 57 (1998), 2906-2913.

[7] G. Taylor, P.G. Saffman. A note on the motion of bubbles in a Hele-Shaw cell and porous medium. Quart. J. Mech. Appl. Math., 12 (1959), 265-279.

[8] T.A. Witten, L.M. Sander. Diffusin-limited aggragation. Phys. Rev., B 27 (1983), 5686-5697.

[9] J. Mathiesen, I. Procaccia, H. L. Swinney, M. Thrasher. The universality class of diffusion-limited aggregation and viscous-limited aggregation. Europhys. Lett., 76 (2006) No. 2, 257-263.

[10] A. Arneodo, Y. Couder, G. Grasseau, V. Hakim, M.Rabaud. Uncovering the analytical Saffman-Taylor finger in unstable viscous fingering and diffusion-limited aggregation. Phys. Rev. Lett., 63 (1989), 984-987.

[11] A. Arneodo, J. Elezgaray, M. Tabard, F. Tallet. Statistical analysis of off-lattice diffusion-limited aggregates in channeland sector geometries. Phys. Rev., E 53 (1996), 6200-6223.

[12] E. Somfai, R.C. Ball, J.P. DeVita, L.M. Sander. Diffusion-limited aggregation in channel geometry. Phys. Rev., E 68 (2003), 020401

[13] M.B. Hastings, L.S. Levitov. Laplacian growth as one-dimensional turbulence. Physica, D 116 (1998), $244-252$.

[14] O. Agam. Viscous fingering in volatile thin films. Phys. Rev., E 79 (2009), 021603.

[15] H. Diamant, O. Agam. Localized Rayleigh instability in evaporation fronts. Phys. Rev. Lett., 104 (2010), 047801.

[16] V.M. Entov, P.I. Étingof. Some exact sdolutions of the thin-sheet stamping problem.Fluid Dyn., 27 (1992), $169-176$.

[17] M. Doi. 2nd quantization representation for classical many-particle system. J. Phys., A 9 (1976), $1465-1477$.

[18] L. Peliti. Path integral approach to birth-death processes on a lattice. J. Physique, 46 (1985), $1469-1483$.

[19] B. P. Lee. Renormalization-group calculation for the reaction $k A \rightarrow \emptyset$. Phys., A 27 (1994), 2633-2652.

[20] J. Cardy, U. C. Tauber. Theory of branching and annihilating random walks Phys. Rev Lett., 77 (1996), $4780-4783$.

[21] Here the Hamiltonian which defines the evolution does not account for the constraint that $A$ particle cannot be born on a site ocuupied by $B$ particle. This constraint can be taken into account by replcing the term $\mu\left(1-a_{j}^{\dagger}\right)$ with $\mu\left(1-a_{j}^{\dagger}\right) \Theta\left(\epsilon-b_{j}^{\dagger} b_{j}\right)$, where $\Theta(x)$ is the haviside function and $\epsilon$ is a positive infintesimal number. 